East African Medical Journal Vol. 80 No. 10 October 2003

COMPARISON BETWEEN SONOGRAPHIC AND PLAIN RADIOGRAPHY IN THE DIAGNOSIS OF SMALL BOWEL OBSTRUCTION AT MULAGO HOSPITAL, UGANDA

F. Musoke, MBChB, MMed (Rad), Senior House Officer, M. G. Kawooya, MBChB, MMed (Rad.), Associate Professor and E. Kiguli-Malwadde, MBChB, MMed (Rad.), Senior Lecturer, Department of Radiology, Makerere University, P.O. Box 7051, Kampala, Uganda

Request for reprints to: Dr. E. Kiguli-Malwadde, Mulago Hospital, P.O. Box 7051, Kampala, Uganda

\title{
COMPARISON BETWEEN SONOGRAPHIC AND PLAIN RADIOGRAPHY IN THE DIAGNOSIS OF SMALL BOWEL OBSTRUCTION AT MULAGO HOSPITAL, UGANDA
}

\author{
F. MUSOKE, M. G. KAWOOYA and E. KIGULI-MALWADDE
}

\begin{abstract}
Objective: To evaluate the role of sonography compared to plain radiography in the diagnosis of patients with suspected small bowel obstruction as well as to determine their specificity, sensitivity and accuracy in the Ugandan setting.

Design: Prospective comparative study.

Setting: Mulago Hospital, Uganda's main referal Hospital and Makerere University Medical School.

Subjects: Seventy patients with suspected small bowel obstruction (SBO) were evaluated at Mulago Hospital between December 1998 and January 2000, 42 patients were males and 28 females. Patients were aged three days to two years and had a clinical suspicion of SBO.

Results: Of the 70 patients, 55 were confirmed to have had SBO. Four had ileus and 11 had no SBO. Sixty four per cent were managed surgically while $36 \%$ were managed conservatively. Sonography made a diagnosis of SBO in $92.7 \%$ and detected strangulation in $71 \%$ of patients. Plain radiography made a diagnosis in $85.5 \%$ of patients and did not detect strangulation. Specificity was $100 \%$ for both, sensitivity $93 \%$, PPV $100 \%$, NPV $73 \%$ for sonography. Sensitivity was $85 \%$, PPV $100 \%$, NPV $58 \%$ for plain radiography.

Main outcome measures: The accuracy of sonography was $93 \%$ as compared to $87 \%$ for plain radiography. The level of obstruction was correctly predicted in $81 \%$ by sonography and $64 \%$ with plain radiography.

Conclusion: It was concluded that sonography is as accurate, specific and sensitive as plain radiography in the diagnosis of SBO as well as determining the level of obstruction in a Ugandan setting. Sonography was found to be more accurate at determining the cause of obstruction and detecting strangulation. It is recommended that sonography should be the initial imaging modality for patients presenting with suspected SBO and plain radiography should only be used as a complimentary investigation.
\end{abstract}

\section{INTRODUCTION}

Small bowel obstruction (SBO) is one of the surgical emergencies that require accurate and prompt diagnosis. At Mulago Hospital, SBO accounts for $40 \%$ of all the cases of intestinal obstruction, which in turn account for $20-30 \%$ of the total annual surgical emergencies (Mulago Hospital register). Common causes of SBO in Uganda differ from those documented in other parts of the world as established from the Mulago hospital register. In Uganda, external hernias form a significant portion of the cases followed by adhesions, in the Western world adhesions followed by neoplasms rank highest $(1,2)$.

At Mulago Hospital, plain radiography is commonly used in evaluating patients suspected to have SBO. Sonography has not been popularly used in evaluating these patients. When clinical assessment points to SBO, a clinician often relies on imaging modalities to further evaluate the patient. Therefore a modality, which accurately and promptly arrives at a diagnosis while preserving the patient's safety and comfort, would be most appropriate. For a long time plain radiography has been used as the initial imaging modality in the evaluation of $\operatorname{SBO}(1,2)$. Plain radiography is sometimes inconclusive or equivocal and rarely detects the cause of obstruction(3). At the same time it cannot assess the presence of strangulation.

Ultrasound has become a popular clinical imaging procedure worldwide(4). Literature suggests that sonography is complimentary to plain radiography in evaluating $\mathrm{SBO}(6-9)$. No comparative data is available for the two imaging modalities at Mulago Hospital; this study attempted to define their value in the diagnosis of SBO at Mulago Hospital. 


\section{MATERIALS AND METHODS}

A prospective comparative study was carried out at Mulago Hospital between December, 1998 and September, 1999. Seventy patients (42 males, 28 females) aged three days to 72 years (mean age 33 years) with a clinical diagnosis of SBO were evaluated using plain radiography and abdominal sonography in the Department of Radiology, Mulago Hospital. The patients selection depended on presence of symptoms compatible with mechanical SBO. These included acute abdominal pain, abdominal distension, vomiting and constipation. The sonographic examination was done prior to plain radiography to avoid bias. The senior house officer under the supervision of a senior radiologist using the available sonoline prima or sonoline SL-1-ultrasound machines performed all the sonographic examinations. Using either 3.5, 5.0 or $7.5 \mathrm{mHz}$ curved or linear array probes. A $7.5 \mathrm{MHz}$ probe is especially useful in very thin patients and children. Sonography was performed with the patients in supine and lateral positions, preferably with a full urinary bladder. All the areas of the abdominal cavity and pelvis were examined using graded compression technique. When the abdomen was "gassy" emphasis was put on the flanks.

At sonography, differentiation of the dilated colon and small bowel loops depended on location and course for the bowel loop as well as absence or presence of vulvulae conniventes for small bowel and haustra for colon. Dilated jejunal and ileal loops were distinguished by analysing the pattern (height and frequency) of the vulvulae conniventes. The loop with high and frequent vulvulae conniventes was considered jejunal and when sparse and low or absent, it was considered ileal.

Mechanical SBO was considered present at sonography, whenever the lumen diameter was greater than $25 \mathrm{~mm}$ for the jejunum and $15 \mathrm{~mm}$ for the ileum over a length of more than $100 \mathrm{~mm}$ or more than three loops together $(1,2)$. Important sonographic signs like increased intraluminal contents (fluid and echoes), increased or pendulous peristalsis of the dilated segment shown by rapid progression or pendulous motion of the bowel contents and collapsed distal segment were considered. The level of obstruction was determined by the location of dilated bowel loops and pattern of vulvulae conniventes at the distally dilated loops as described by Ko (1). Where an obvious abnormality coincided with the transition point e.g. an (abscess, cyst or intussusception) it was considered the cause of obstruction(1). Where no apparent cause at the transitional zone was seen; peritoneal fibrous adhesions were considered the cause of obstruction. This is referred to as the sonographic criteria for adhesion. Sonographic signs of strangulation and gangrene like localised and generalised ascites, aperistaltic loops, air in portal vein and intra luminal fluid-fluid levels were also assessed.

Paralytic ileus was considered present when signs like dilated aperistaltic SB loops with abundant gas and dilated colon filled with gas, fluid or stool were noted. Sonographic results were analysed on basis of the initial report at the time of examination. Hard copy sonograms were made for record. Two senior radiologists did the image interpretation. The major draw back with the use of ultrasound is that it is operator dependant. This may be a cause of error, that's why two radiologists worked with the senior house officer to overcome this.
After sonography supine and erect plain abdominal Xrays were done on all patients. The lateral decubitus film was not done because of financial constraints. The radiological findings, clinical and laboratory data were entered into a data sheet. The clinical final diagnosis at discharge was considered the standard of reference. This was obtained at surgery, histology or by the patient clinical course. This was established at surgery in 35 patients and by clinical evaluation in 35 patients.

\section{RESULTS}

Seventy patients from different parts of Uganda were evaluated. The age range was three days to 72 years (mean age 33 years), $40 \%$ were females, $60 \%$ were males (Figure 1). Fifty five patients were confirmed to have mechanical SBO. Four had ileus while eleven had no SBO. Of the 55 patients, 35(64\%) were managed surgically, 20 (36\%) were managed conservatively. All the 55 patients, who had mechanical SBO had pendulous type of peristalsis and intraluminal fluid with echoes. Jejunal diameter ranged between 25$40 \mathrm{~mm}$ while ileal diameters ranged between $15 \mathrm{~mm}$ $25 \mathrm{~mm}$. Sonography made a diagnosis of SBO in 51 patients $(92.7 \%)$ while it predicted the level of obstruction in 29 of surgically proven 35 cases (83\%). The cause was detected sonographically in 20 out of 55 cases (36\%) (Tables 1 and 2). When the sonographic criterion for adhesions was used this increased to 44 out of 55 cases $(80 \%)$. Sonography detected seven of the nine patients with strangulation $(77 \%)$. All these seven were proved surgically and all had intraluminal fluid-fluid levels at sonography. None of these patients were detected by plain radiography.

Table 1

Results of sonographic examination for small bowel obstruction

\begin{tabular}{llll}
\hline Causes of obstruction & $\begin{array}{l}\text { No. of } \\
\text { cases }\end{array}$ & $\begin{array}{l}\text { Obstruction } \\
\text { diagnosed }\end{array}$ & $\begin{array}{l}\text { Cause } \\
\text { detected }\end{array}$ \\
\hline Adhesions & 24 & 24 & 0 \\
External hernia & 18 & 14 & 14 \\
Appendicular mass & 2 & 2 & 2 \\
Appendicular abscesses & 1 & 1 & 1 \\
Ovarian cyst & 2 & 2 & 2 \\
SB Volvulus & 1 & 1 & 0 \\
Internal hernia & 2 & 2 & 0 \\
Ileal atresia & 2 & 2 & 0 \\
Ilio-sigmoid knotting & 2 & 2 & 0 \\
Intussusception & 1 & 1 & 1 \\
\hline Total & 55 & 51 & 20 \\
\hline
\end{tabular}

Plain radiographic examination made a diagnosis in 47 patients $(85.5 \%)$. The cause of obstruction was only detected in six cases $(11 \%)$. 
Table 2

Results of radiography examination for small bowel obstruction

\begin{tabular}{llll}
\hline Causes of obstruction & $\begin{array}{l}\text { No. of } \\
\text { Cases }\end{array}$ & $\begin{array}{l}\text { Obstruction } \\
\text { diagnosed }\end{array}$ & $\begin{array}{l}\text { Cause } \\
\text { detected }\end{array}$ \\
\hline Adhesions & 24 & 23 & 0 \\
External hernia & 18 & 11 & 6 \\
Appendicular mass & 2 & 2 & 0 \\
Appendicular abscess & 1 & 1 & 0 \\
Ovarian cyst & 2 & 2 & 0 \\
SB volvulus & 1 & 1 & 0 \\
Internal hernia & 2 & 2 & 0 \\
Ileal atresia & 2 & 2 & 0 \\
Ilio sigmoid knotting & 2 & 2 & 0 \\
Intussusception & 1 & 1 & 0 \\
\hline Total & 55 & 47 & 6 \\
\hline
\end{tabular}

Specificity was $100 \%$ for both, sensitivity $93 \%$, PPV 100\%, NPV 73\% for sonography. Sensitivity was $85 \%$, PPV 100\%, NPV $58 \%$ for plain radiography. Statistical analysis using the chi square test showed that there was no significant difference between the results of the two diagnostic modalities in making a diagnosis of SBO and determining the level of obstruction (P>0.5) at 5\% level of confidence limits. There was significant difference between sonography and plain radiography $(\mathrm{P}>0.05)$ at $5 \%$ level of confidence at detection of the cause of SBO.

Figure 1

Patients distribution by age

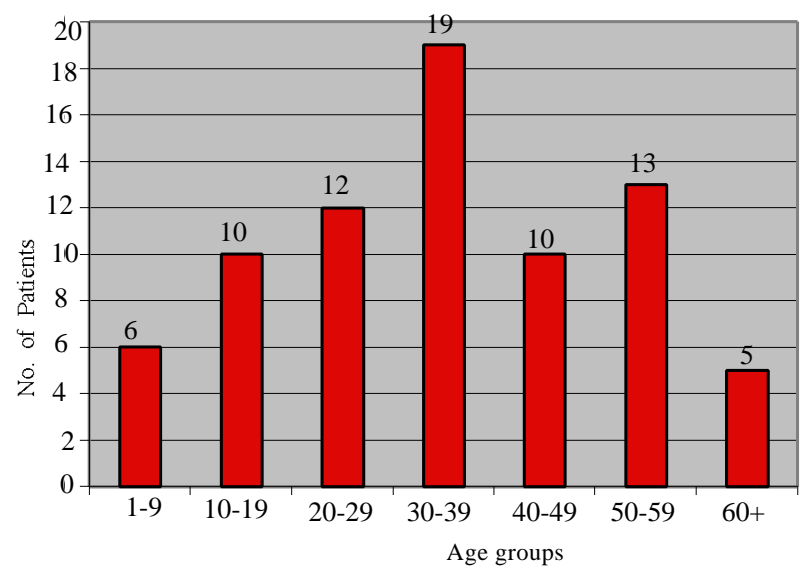

Patients less than one year are included in the 1-9 group.
Figure 2a

Longitudinal scan through the lower quadrant demonstrates a dilated SOB loop (ileum) and a thick walled cystic mass posterior to the urinary bladder with internal echoes. The distally dilated ileum tapers off at the level of the mass

\section{Figure 2b}

Transverse scan. The thick walled round cystic mass is noted in the recto vesicle pouch

Sonographic examination using a $5.0 \mathrm{MHz}$ curved array probe

Figure 2c

Plain abdominal film demonstrates non-specific gaseous shadows with no dilated SB loops 
Images of a 36-year-old male who was lame since childhood and had scoliosis because of the lower limb deformity. He was referred with features suggestive of intestinal obstruction. He had been treated conservatively for appendicitis. Sonographic examination using a 5.0 $\mathrm{MHz}$ curved array probe was done.

\section{Figure 3a}

Longitudinal scan through the right iliac region demonstrates an echo complex mass at the transitional point of the distally dilated ileal loop. The level of obstruction is at the ileum

\section{Figure 3b}

Longitudinal scan demonstrates multiple dilated jejunal loops with increased intra luminal fluid and echoes. This was an appendicular mass

\section{Figure 3c}

Supine film reveals multiple gaseous dilated SB loops

\section{Figure 3d}

Erect film shows multiple SB air-fluid levels on the right with no obvious mass seen

The images of a 35-year old female with history of salpingoophrectomy six months ago

\section{Figure 4a}

Longitudinal scan of the abdomen depicts multiple fluidfilled dilated ileal loops with intra luminal echoes and a little inter loop fluid collection. No cause detected

\section{Figure 4b}

Transverse and oblique scans through the lower quadrant reveals intraluminal fluid-fluid levels 


\section{Figure 4c}

Erect film demonstrates multiple air fluid levels with a "string of beads" sign. No sign of strangulation

Plain radiographs of abdomen reveal scoliosis with concavity to the right.

\section{Figure 4d}

Supine film demonstrates gaseous dilated jejunal loops with no signs of strangulation

Plain radiograph of the abdomen.

\section{DISCUSSION}

The usefulness of sonography in the evaluation of SBO has been documented in a few previous studies $(1,2,5)$. In Mulago Hospital, plain radiography is widely used for investigating patients with suspected SBO. This is because it is the examination that is widely accepted by the doctors. Plain radiography has been used for along time and has been found to be fairly accurate. No studies showing the advantages of ultrasound had been done before in Mulago Hospital so there was no need for change. In this study, we were able to establish that sonography, which has not been popularly used in evaluating SBO at Mulago Hospital is as useful as plain radiography in confirming the diagnosis and defining the level of obstruction as well as identifying the complication of strangulation. We feel that these results apply to Uganda as a whole because the patients studied were from different regions of the country, of both sexes and of varying ages. There was no statistical difference between the specificity, sensitivity and accuracy of both imaging techniques. This is similar to what has been reported in previous studies. These findings are significant in the developing world because sonography is cheap, portable and does not use ionising radiation. This is particularly significant for Mulago Hospital as trained manpower and ultrasound equipment is now available. The patients with suspected SBO are usually, very sick and so need quick and portable means of investigation like ultrasound. Schmutz et al(1), had earlier reported that a gassy abdomen was a significant set-back in evaluation of patients with SBO, however this was not the case in the study. A gassy abdomen was only encountered in one patient. This could be because of the differences in diet between the populations.

The commonest cause of SBO in our study were adhesions $44 \%$ and external hernias $33 \%$ compared to $22 \%$ adhesions and neoplasms $24 \%$ in Western studies as in Schmutz et al report(1). In Japan adhesions account for $64 \%$ of the causes of SBO(5). Adhesions are common causes of SBO in Uganda and can be indirectly diagnosed using sonography whereas this is not possible with plain radiography. There is an increase in adhesions as a cause of SBO in Uganda as earlier reported by S. Wandira who did work on intestinal obstruction at Mulago Hospital in 1997 (unpublished). The number of external hernias is falling possibly because of increased awareness amongst the population as well as improved surgical facilities. The improved surgical facilities further explain the increase in surgery, which would explain the increase in adhesions. All the cases with adhesions had a previous surgery. Inguinal hernias were the major external hernias in this study. This has not been the case in Western world studies. Why they are common in our series and not in the Western world remains to be studied.

Adhesions formed a large proportion of the aetiologies in the Ko et al(2) study but he did not use the sonographic criteria for diagnosis of adhesions so the value of detected aetiologies was low. Four patients in the study detected to have adhesions using the sonographic criteria were proved to have SBO due to adhesion surgically because they had strangulation. Although the number in this study is small, we feel that this criterion is useful. Complicated SBO (Strangulation) was found in nine patients at surgery. This is high compared to only one patient in Ko et al. series (2). This possibly results from a delay in reporting to hospital and is easily explained by low social economic status, limited hospital facilities, poor means of transport and lower doctor patient ratio in the developing countries.

Sonography was found to be better than plain radiograph at establishing the diagnosis of SBO and differentiating between paralytic ileus and mechanical obstruction. In conclusion, sonography where expertise and equipment is available should be the initial investigation in patients presenting with SBO and that plain radiography should only be used as a complementary tool. 


\section{REFERENCES}

1. Schmutz, G. R., Benko, A., Rourier, L., et al. Small bowel obstruction. Role and contribution of sonography. Eur. Radiology. 1997; 7:1054 -1058.

2. Ko, Y.T., Lim, J.H., Lee, H.W., and Lim, J. W. Small bowel obstruction. Sonographic evaluation. Radiology. 1993; 188:649653.

3. Mc Adam. A three-year review of intestinal obstruction at Mulago Hospital Kampala Uganda. East. Afr. Med. J. 1961; 38:536-544.

4. C.R. Hill. History-Now and then clinical ultrasound. A comprehensive text Abdominal and general Ultrasound. VI 1993; 1:3-10.

5. Meiser, G., and Meissner, K. Sonographic differential diagnosis of intestinal obstruction: Results of prospective study of 48 patients. Ultraschall Med. 1985; 6:39-45.

6. Maglinte, D.D.T., Emil, J., Balthazar, E.J., Fredrick M.K. et $a l$. The role of radiology in the diagnosis of small bowel obstruction. Amer. J. Radiology. 1997; 168:179-186.

7. Julien, B.C.M., Puyleart F.M., Van der Zan, and Eric M.R, Sonography and the acute abdomen: Practical consideration. Amer. J. Radiology. 1997; 179-186.

8. Ogata, W., Mai, S., Hosotani, R., et al. Abdominal ultrasound for the diagnosis of strangulation in small bowel obstruction. Brit. J Surg. 1994; 81:421-424.

9. Di, Mizio, R., Grassi, R., Marchese, E., Basti M., et al. Uncomplicated small bowel obstruction in adults. Ultrasonographic findings of fluid between loops and its prognostic value. Radio Med Toriono. 1995; 89:487-491.

\section{ADVERTISEMENT}

\section{NEW COLDCAP PRESENTATIONS}

Regal Pharmaceuticals Ltd., one of the leading local manufacturers of pharmaceutical products, has developed two new cold and flu preparations that will complement Coldcap Capsules and Coldcap Syrup, which have been in the market for over ten vears.

Coldcap Day Time and Coldcap Night Time are intended for use by those who do not wish to have their daily routines disrupted by cold and flu remedies.

Coldcap Day Time has been specifically formulated to provide relief for people who have busy lifestyles and people who cannot afford to fall drowsy during the day.

It contains Paracetamol BP 500mg, Pseudoephedrine Hydrochloride BP 30mg and Caffeine Anhdrous BP $30 \mathrm{mg}$.

It's available in a hygienic blister pack of 12 capsules which retails at a price of Kshs.89/= a pack.

Coldcap Night Time is suitable for the rapid relief of symptoms of cold and flu at night when a peaceful night's sleep is essential.

It contains Paracetamol BP 500mg and Chlorpheniramine Maleate BP 2mg.

It is also available at a retail price of Kshs.89/= per hygienic blister pack on 12 capsules.

The company says that the addition of Coldcap Day Time and Coldcap Night Time to the existing two formulations, gives those suffering from colds and flu an opportunity to choose the product that is suited to them and their circumstances.

- $\quad$ The range is conveniently colour coded for easy identification.

- The original Coldcap Capsules now come in a new blister pack of 12 capsules that carries more product information and the wording "Original". The pack is smaller and consistent with the new presentations, and easily slips into the pocket.

- The entire Coldcap range can be obtained with or without a prescription from authorized pharmacies only, as they require the input of a heathcare provider.

(3) REGAL PHARMACEUTICALS LTD. P.O. BOX 44421, NAIROBI 